\title{
Urabstimmung FMH Services 2010
}

\author{
Max Giger ${ }^{a}$, Reinhard Kunz \\ a Dr. med., \\ Präsident FMH Services \\ b Geschäftsführer \\ FMH Services
}

Korrespondenz: FMH Services

Burghöhe 1

CH-6208 Oberkirch

Tel. 0419250077

mail@fmhservices.ch
Wir freuen uns, Sie über das Geschäftsjahr 2009 informieren zu dürfen. Einmal mehr können wir Ihnen einen erfreulichen Abschluss präsentieren. Dieser resultiert aus der breiten Akzeptanz unserer Dienstleistungen und der Erholung der Finanzmärkte. Im Geschäftsbericht bieten wir Ihnen einen Überblick unserer Dienstleistungen und legen unsere Gedanken dar zum Geschäftsjahr und zur unternehmerischen Entwicklung der Praxiswelt. Die solide Eigenkapitalbasis erlaubt uns, die Dienstleistungen weiter auszubauen und Sie 2010 in wichtigen Fragen zu unterstützen.

Im vergangenen Jahr haben wir unsere Geschäftsbasis weiter ausgebaut. Unsere Internetplattformen und Dienstleistungen stossen auf ein grosses Inter- esse. Unsere weitgehend unentgeltliche Unterstützung mit Beratung, Seminaren und Fachschriften zu aktuellen Themen wird sehr rege genutzt.

Mit Ihrem Beitritt zur FMH sind Sie auch als Mitglied in der Genossenschaft FMH Services aufgenommen worden. Wir zählen heute über 30000 Mitglieder. Wir möchten Sie bitten, an der Urabstimmung 2010 teilzunehmen. Die erforderlichen Dokumente finden Sie in dieser Ausgabe der Schweizerischen Ärztezeitung. Mit Ihrer Teilnahme können Sie den Geschäftsbericht und die Rechnung 2009 genehmigen, die Verwaltung entlasten und die Abwicklungs- und Revisionsstelle wählen.

Wir danken Ihnen schon heute für Ihr Interesse und Ihren Einsatz.

\section{Votation générale de FMH Services 2010}

\author{
Max Giger ${ }^{a}$, Reinhard Kunz ${ }^{b}$ \\ a Dr, président \\ de FMH Services \\ b Directeur de FMH Services
}

C'est avec plaisir que nous vous informons du bilan positif pour l'exercice 2009. Ce réjouissant résultat est dû au large succès de nos prestations et au rétablissement des marchés financiers. Le rapport de gestion vous donnera un aperçu de nos services. Il vous présentera également nos réflexions sur l'exercice écoulé et sur l'évolution du marché des cabinets médicaux. Grâce à un solide capital propre, nous pourrons continuer à enrichir notre palette de prestations en 2010 et à vous épauler dans toutes les questions importantes auxquelles vous serez confrontés.
En adhérant à la FMH, vous êtes automatiquement affilié à la coopérative FMH Services qui compte aujourd'hui plus de 30000 membres. Nous vous invitons à participer à la votation générale 2010 . Vous trouverez les documents nécessaires dans la présente édition du Bulletin des médecins suisses. Vous êtes appelés à approuver le rapport de gestion et les comptes 2009, à donner décharge à l'administration et à élire l'organe de révision.

En vous remerciant d'ores et déjà de votre intérêt et de votre participation, nous vous présentons, chères associées, chers associés, nos salutations les meilleures.

\footnotetext{
L'exercice écoulé nous a permis d'étendre notre offre. Nos plates-formes internet, ainsi que nos conseils majoritairement gratuits, séminaires et publications spécialisées sur des sujets d'actualités rencontrent un franc succès.

Correspondance:

Burghöhe 1

CH-6208 Oberkirch

Tél. 0419250077

mail@fmhservices.ch
} 